\title{
LA FRANCOPHONIE EUROPÉENNE ET TCHÈQUE CENT TRENTE ANS APRÈS L'INVENTION DU TERME PAR ONÉSIME RECLUS
}

\author{
Zuzana Raková
}

\begin{abstract}
In assessing statistical sources, the article compares the state of the European and global Francophonie in 1880 and at the present time. The European Francophonie, or rather the Francophonies, because there are countries with different status within the Francophonie, is special compared to other continents. At the bottom of a historical study and evaluation of statistical data, the article aims to respond primarily to the following questions: what is the paradox of the current European Francophonie? What is the specificity of the French-speaking Czech compared to other European countries? How to promote policy for multilingualism in the concrete situation of the Czech Republic?

Keywords: Onesimus Reclus; International Organization of the Francophonie; teaching of French; native French speakers, partial French speakers partial.

Résumé : En évaluant des sources statistiques, l'article compare l'état de la francophonie européenne et mondiale en 1880 et à l'époque actuelle. La francophonie européenne, ou plutôt les francophonies, parce qu'il y a des pays ayant les statuts variés au sein de la Francophonie, est particulière par rapport à celle des autres continents. Au fond d'une étude historique et d'une évaluation des données statistiques, l'article a pour objectif de répondre avant tout aux questions suivantes : en quoi consiste le paradoxe de la francophonie européenne actuelle ? Quelle est la spécificité de la francophonie tchèque par rapport à d'autres pays européens ? Comment promouvoir la politique pour le plurilinguisme dans la situation concrète de la République tchèque?
\end{abstract}

Mots clés : Onésime Reclus ; Organisation internationale de la Francophonie ; enseignement du français : locuteurs francophones natifs; locuteurs francophones partiels.

\section{Introduction}

Le géographe Onésime Reclus (1837-1916) est généralement connu comme l'inventeur du terme francophonie, qu'il emploie pour la première fois vers 1880, dans le cadre de sa réflexion sur le destin colonial français. Dans son ouvrage France, Algérie et colonies, il définit les francophones comme « tous ceux qui sont ou semblent être destinés à rester ou à devenir participants de notre langue ». Le facteur linguistique est essentiel pour lui comme critère de définition du terme.

Dans l'ouvrage mentionné, O. Reclus estime à 47825000 personnes la population probable au 31 décembre 1880 des francophones dans le monde : 41600000 en Europe, 
3560000 en Afrique, 2580000 en Amérique et 85000 en Asie. Dans Le plus beau royaume sous le ciel, Onésime Reclus dénombre à la fin du XIX ${ }^{\mathrm{e}}$ siècle près de 43 millions de francophones en Europe, moins de 4 millions en Amérique, et à peu près 8 millions en Afrique, ce qui nous mène à 54 ou 55 millions (voir graphique $\mathrm{n}^{\circ} 1$ ).

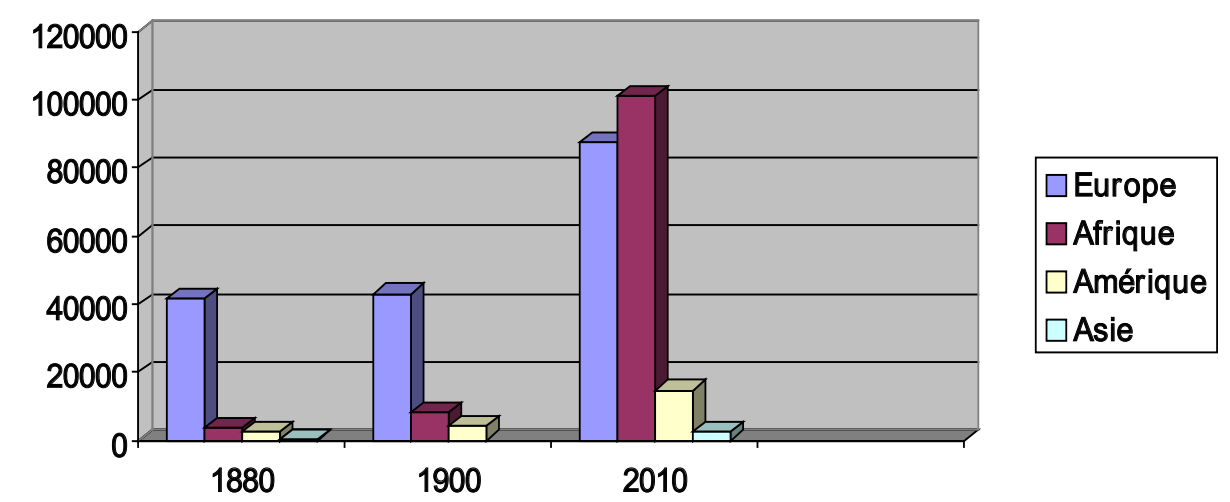

Graphique $n^{\circ} 1$ : Nombre absolu (en milliers) de locuteurs francophones sur les quatre continents en 1880, 1900 et 2010.

Sources : http:/ / www.ladocumentationfrancaise.fr/dossiers/francophonie/ onesime-reclus.shtml, le 30/07/2011; La Langue française dans le monde 2010, Paris : Nathan, 2010. pp. 11, 14-15, 130-131, 154-155.

\section{La Francophonie modiale et européenne actuelle}

Qu'en est-il de la francophonie en Europe et dans le monde plus de cent trente ans après la parution de l'œuvre optimiste d'Onésime Reclus?

Arrêtons-nous un moment sur les chiffres récents, apportés par les auteurs de l'Atlas mondial de la francophonie (2006) et ceux de La Langue française dans le monde 2010.

Selon l'Atlas mondial de la francophonie, il y avait 175 millions de locuteurs francophones dans le monde, au 1er janvier 2006. À cette date, 53 États et gouvernements membres et 10 pays observateurs composaient l'Organisation internationale de la francophonie. L'Europe de l'Ouest avait 73 millions de locuteurs francophones, l'Europe centrale et les Balkans comptaient ensemble 6,5 milliones de francophones, l'Afrique du Nord et l'Afrique subsaharienne totalisaient 70 millions de francophones.

L'élargissement institutionnel de la Francophonie continue avec succès, puisqu'elle comprend 75 États et gouvernements (56 membres et 19 observateurs) dès l'octobre 2010. Il y aurait à cette date 220 millions de francophones - soit une progression de 20 millions par rapport à 2007 (200 millions). Et les auteurs de l'ouvrage La Langue française dans le monde 2010 estiment même qu'en 2050, les locuteurs francophones pourraient dépasser les 700 millions dont 600 millions vivraient en Afrique, 80 millions environ en Europe et le reste en Amérique, en Asie et en Océanie. On nous apprend qu'avec au minimum 220 millions de francophones, le français ne fut jamais autant parlé dans le monde qu'à l'heure actuelle. L'œuvre nous fait découvrir également qu'avec au moins 
116 millions d'apprenants, le français est l'une des langues les plus enseignées dans le monde, même si le choix d'une seule langue étrangère qui tend à s'imposer dans les systèmes scolaires de plusieurs pays européens menace son avenir. Malgré cela, les auteurs gardent une vision assez optimiste quant à l'avenir du français dont ils estiment le nombre de locuteurs jusqu'à 700 millions d'ici 2050. Le continent ayant le potentiel le plus grand pour l'augmentation du nombre de francophone est l'Afrique qui pourrait compter à elle seule 500 millions de locuteurs francophones en 2050, tandis que les effectifs des francophones sur les autres continents devraient rester stables selon les estimations des auteurs. L'Europe devrait compter en 2050 les 85 millions de francophones qu'elle a aujourd'hui, mais le poids relatif de la francophonie européenne diminuerait sensiblement puisque ce chiffre représenterait seulement $12 \%$ des francophones de la planète tandis qu'en 2010, l'Europe totalisait $40 \%$ des francophones du monde.

Abandonnons les estimations dont seul l'avenir peut confirmer ou infirmer le bienfondé et concentrons-nous sur l'analyse de la situation actuelle de la francophonie mondiale. Selon les auteurs de La Langue française dans le monde 2010, le nombre de locuteurs francophones et francophones partiels vivant dans les pays de l'OIF en 2010 fut 2.564.500 en Asie et Océanie, 100.952.200 sur le continent africain, 87.498.900 en Europe et 14.717.000 en Amérique, y compris les Caraïbes. Les francophones de tous les pays de l'Organisation internationale de la Francophonie avaient été donc au moins 205.732.600 dans le monde entier en 2010, si nous additionnons les statistiques données séparément pour chaque pays de l'OIF. Les 220 millions de locuteurs francophones dont les auteurs parlent dans le texte s'expliquent par le fait que les tableaux statistiques apportent uniquement les données sur les locuteurs francophones ou francophones partiels des pays appartenant à l'OIF. Nous pouvons donc supposer qu'il y avait 14.267.400 des francophones dans le monde en 2010 hors l'OIF, soit 6,5\% des francophones du monde vivant hors la Francophonie institutionnelle (voir graphique $\mathrm{n}^{\mathrm{o}} 1$ ).

Il est non moins intéressant de constater l'importance relative des locuteurs francophones des pays de l'OIF sur la population totale de chaque continent. Ainsi, le continent le moins francophone est sans aucun doute l'Asie, avec seulement 0,06 \%, vient ensuite l'Amérique où les francophones représentent $1,55 \%$ de la population des deux continents, l'Afrique avec 9,56 \% des locuteurs francophones et l'Europe qui, en chiffres relatifs, apparaît comme le continent le plus francophone puisqu' elle compte 11,93 \% des francophones. Il s'agit cependant des chiffres sous-estimés car ne sont pas dénombrés les locuteurs francophones des pays n'appartenant pas à l'OIF comme l'Algérie, les ÉtatsUnis ou l'Israël. Nous disposons cependant des statistiques fiables pour ces pays, mais celles-ci ne datent pas de 2010, mais de 2008 pour l'Algérie (11.200.000 de francophones) et l'Israël (500.000 francophones), de 2001 pour Val d'Aoste (90.000 francophones) et de 2000 pour les États-Unis (2.100.000 de francophones ou francophones partiels). Les francophones africains des pays de l'OIF et de l'Algérie et de l'Israël représentent donc $10,66 \%$ de la population africaine (112.652.200 de francophones sur 1.056.527.000 d'habitants). Les données pour les pays francophones européens de l'OIF peuvent être complétés par les francophones du Val d'Aoste, ce qui nous mène à 87.588.900, soit 11,94 \% de francophones sur la population totale de l'Europe. En Amérique, si nous comptions également les francophones vivant aux États-Unis, nous aboutirions à 16.817 .000 de francophones ou francophones partiels sur le continent américain, soit 1,77 \% de la population totale. 
Cependant, ces chiffres occultent une réalité évidente : il y a aussi pas mal de locuteurs francophones dans le monde qui vivent hors les pays de l'OIF et hors l'Algérie, l'Israël, le Val d'Aoste et les États-Unis.

C'est pourquoi il n'est pas sans intérêt de rendre compte aussi des taux de francophonie réelle dans les pays de l'OIF de chaque continent, ce qui signifie le nombre de locuteurs francophones dans les pays de l'OIF par rapport à la population totale vivant dans les pays organisés dans cette organisation sur chaque continent (voir graphique $\mathrm{n}^{\circ} 2$ ).

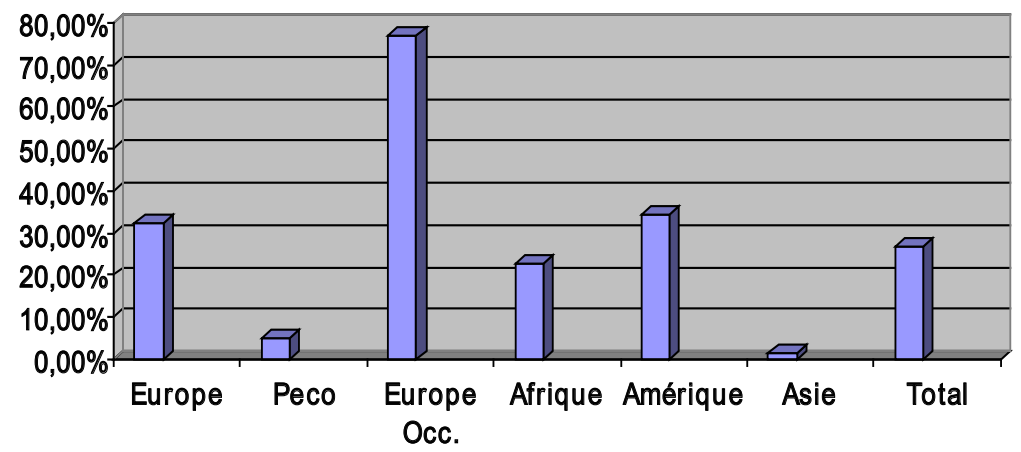

Graphique $n^{\circ} 2$ : Le taux de francophonie réelle au sein de 1'OIF sur les 4 continents en 2010 (proportion des locuteurs francophones /francophones partiels sur la population totale dans les pays membres de l'OIF)

Sources: La Langue française dans le monde 2010, Paris : Nathan, 2010, pp. 11, 14-15, 130-131, 154-155.

On voit clairement que le taux de francophonie réelle le plus faible est celui de l'Asie et de l'Océanie, dont les locuteurs francophones et francophones partiels ne représentent que $1,37 \%$ des habitants vivant en pays asiatiques de l'OIF, soit 2.564 .500 de locuteurs francophones / francophones partiels sur les 186.753 .000 d'habitants. Vient ensuite l'Afrique dont les locuteurs francophones / francophones partiels vivant dans l'OIF sont au nombre de 100.952 .200 sur les 443.843 .000 d'habitants, soit 22,7 \%. Le taux le plus fort de francophonie dans le cadre des pays de l'OIF ont l'Amérique avec 34,3\%, soit 14.717.000 de locuteurs francophones / francophones partiels sur les $42.920 .900 \mathrm{~d}$ 'habitants vivant en l'OIF, et l'Europe avec 32,34 \%, en chiffres absolus 87.498 .900 de locuteurs francophones sur 270.525.000 d'habitants vivant en l'OIF. Globalement, les locuteurs francophones / francophones partiels des pays de l'OIF totalisent 205.732.600 sur un ensemble de 761.015.800 d'habitants de ces pays. Le taux de «francophonie réelle», c'està-dire le pourcentage de locuteurs francophones ou au moins de francophones partiels sur l'ensemble de la population vivant en pays qui adhèrent à l'OIF, sur les quatre continents ensemble - l'Australie étant comprise parmi les pays de l'Océanie - est de $27 \%$. Seulement un peu plus d'un quart de la population mondiale habitant dans les pays de l'OIF est capable de s'exprimer et d'écrire en français, et encore à un niveau extrêmement variable.

\section{Le paradoxe de la Francophonie européenne}

La francophonie européenne, ou plutôt les francophonies, parce qu'il y a des pays ayant les statuts variés au sein de la Francophonie, est un peu particulière par rapport aux 
autres continents. Il faut noter que la francophonie européenne actuelle est assez paradoxale. Son paradoxe consiste notamment dans le fait que beaucoup de pays européens sont organisés dans l'Organisation internationale de la Francophonie, mais l'Europe dans son ensemble n'a qu'un nombre faible de francophones réels et elle a encore moins de francophones natifs.

Ceci est vrai notamment des pays de l'Europe centrale et orientale (Peco) de l'OIF au sein desquels les francophones et les francophones partiels ne constituent que 5,3\% de la population (8.932.143 d'habitants francophones sur un total de $168.531 .000 \mathrm{~d}$ 'habitants). Les francophones réels sont par contre beaucoup plus nombreux dans les pays de l'OIF de l'Europe occidentale où le taux de francophonie (ou de locuteurs francophones) est de $77 \%$ (78.535.380 de locuteurs francophones parmi les $101.994 .000 \mathrm{~d}$ 'habitants). Les statistiques montrent donc clairement que les pays européens ayant adhéré à l'OIF récemment, à partir des années 1990, ont des pourcentages de population francophone en-dessous de la moyenne dans le cadre de l'OIF, tandis que les pays traditionnellement francophones de l'Europe occidentale, appartenant à la Francophonie soit dès le début en 1970, soit dès les années 1970 ou 1980, ont des nombres relatifs de locuteurs francophones très en-dessus de la moyenne mondiale. Il s'agit surtout des pays tels que la France, la Suisse, la Belgique, le Monaco, dont les locuteurs francophones sont en grande partie des francophones natifs, à la différence des Peco, où la francophonie est presque uniquement acquise par l'apprentissage scolaire sur tous les niveaux, de l'enseignement primaire et secondaire jusqu'au supérieur. Le français dans ces pays n'a aucun statut officiel, il est seulement langue étrangère ou tout au plus langue d'enseignement dans les établissements français ou bilingues.

C'est pourquoi Robert Chaudenson $(2006$ : 75, 108) déjà en 2006, mettait en garde contre le fait que l'Organisation Internationale de la Francophonie devenait de moins en moins francophone puisque «les États qui y adhéraient n'avaient que des effectifs très faibles de vrais locuteurs du français.» Ce constat est valable pour tous les "pays observateurs» dans l'OIF, y compris pour la République tchèque.

Mais, et c'est une réalité très encourageante pour l'avenir du français en Europe, tandis qu'un grand nombre de pays européens qui font partie de l'OIF n'ont que des effectifs assez faibles de francophones, de l'autre côté plusieurs pays européens n'étant pas organisés dans l'OIF ont des potentiels forts en nombre d'apprenants du français. Par exemple les pays tels que l'Allemagne, l'Italie, l'Espagne, le Portugal, les Pays-Bas, le Royaume-Uni, l'Irlande, la Russie, la Suède, dont aucun n'appartient à l'OIF, ont tous des effectifs d'apprenants du et en français supérieurs à ceux de la République tchèque (qui est membre observateur de l'OIF depuis 1999). Et cela non seulement en chiffres absolus, mais aussi, sauf le Royaume-Unis et la Russie, en chiffres relatifs. Ainsi, l'Allemagne dénombre 1.717.988 d'apprenants du et en français, ce qui représente 2,1 \% de la population totale allemande, l'Italie compte 2.040.519 d'apprenants du et en français, soit 3,4\% de la population du pays, l'Espagne comprend 971.031 apprenants du et en français, ce qui représente $2,1 \%$ de la population espagnole et le Portugal totalise 392.691 apprenants du et en français, ce qui représente 3,7 \% de la population portugaise. Les Pays-Bas dénombrent 403.560 apprenants du et en français, soit 2,4 \% de la population totale, le Royaume-Uni a 187.970 apprenants du et en français, donc 0,3\% seulement de la population britannique, l'Irlande comprend 206.079 apprenants du et en français qui représentent $4,7 \%$ de la population irlandaise. La Russie totalise 754.767 apprenants du 
et en français, soit $0,5 \%$ de la population, et la Suède dénombre 140.500 apprenants du et en français, ce qui représente $1,5 \%$ de la population du pays. Bref, il en résulte que la République tchèque, avec ses 53.863 apprenants du et en français (en 2008-2009), représentant $0,5 \%$ de la population totale, reste loin derrière la plupart des pays non membres de l'OIF de l'Europe occidentale et peut se comparer, en nombres relatifs d'apprenants du français, avec la Russie, qui n'est pas membre de l'OIF, à la différence de la République Tchèque (voir graphiques $\mathrm{n}^{\circ} 3$ et $\mathrm{n}^{\circ} 4$ ).

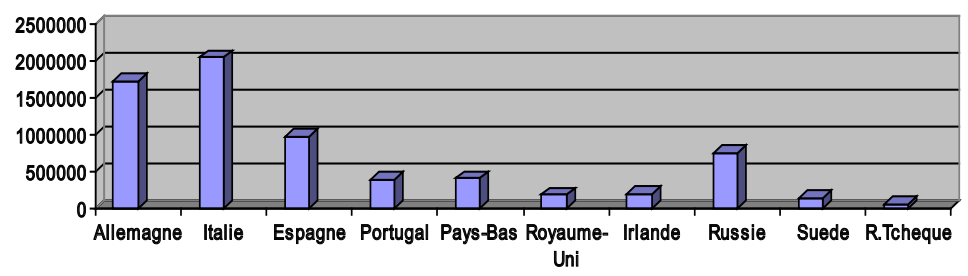

Graphique $n^{0} 3$ : Apprenants du et en français dans plusieurs pays de l'Europe occidentale (pays non membres de l'OIF + la Rép. Tchèque) en 2008-2009, en chiffres absolus Sources : La Langue française dans le monde 2010, Paris : Nathan, 2010, pp. 154-155.

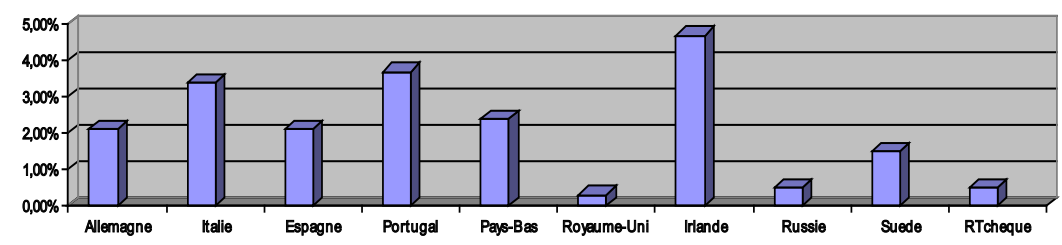

Graphique $n^{\circ} 4$ : Apprenants du et en français dans plusieurs pays de l'Europe occidentale (pays non membres de 1'OIF + la Rép. Tchèque) en 2008-2009, en chiffres relatifs sur la population totale

Sources : La Langue française dans le monde 2010, Paris : Nathan, 2010, pp. 154-155.

\section{Les spécificités de la francophonie centreuropéenne}

Quelle est la cause de cette francophonie réelle plus forte en plusieurs pays européens non membres de l'OIF par rapport à la République tchèque (mais aussi par rapport à la Slovaquie, la Pologne, l'Hongrie, la Littuanie, la Lettonie et d'autres pays des Peco, observateurs de l'OIF) ? La République tchèque serait-elle un mauvais élève dans la famille francophone où sa situation est-elle symptomatique et généralisable à l'ensemble des membres de l'OIF issus des Peco?

La réponse peut être donnée si nous examinons les évolutions du système scolaire tchèque et les causes de la diffusion de telle ou telle langue, dans différentes époques, comme langue étrangère enseignée.

$\mathrm{Au} \mathrm{XIX}^{\mathrm{e}}$ siècle, dans le milieu tchèque, prédominait longtemps la question de la seconde langue territoriale obligatoire dans le système scolaire (la querelle de l'allemand et du tchèque), et ce au détriment d'une autre langue étrangère. Tandis que dans plusieurs pays européens, les langues vivantes, dont en premier lieu le français en tant que langue de la noblesse, de la diplomatie, de la communication internationnale, langue de 
la littérature et de tout individu cultivé, commençaient à s'imposer dans les systèmes scolaires nationaux, les Pays tchèques restaient longtemps à l'écart de ce mouvement. Un facteur important qui en fut cause était le rôle de la langue allemande qui remplissait ces fonctions de langue de culture, de la science, de la politique, bref de langue véhiculaire au sein de l'empire autrichien. Tandis qu'en Prusse par exemple, le français était la première langue vivante étrangère, en Autriche, c'était l'allemand. Ce n'est que dans les années 1860, après que la seconde langue territoriale fut stipulée non obligatoire en Bohême que la voie était ouverte à l'introduction d'une autre langue vivante dans les écoles tchèques et allemandes de Bohême et de Moravie. Ainsi, l'apprentissage du français commençait à se répandre plus massivement à partir des années 1860-1870. Entre 1867-1874, il était introduit comme matière obligatoire dans les programmes scolaires de certains types d'écoles secondaires en Autriche (écoles techniques, lycées techniques, écoles supérieures de jeunes filles, académies de commerce) (Reznikow, 2002 : 535-549).

La francophonie de la population tchèque avait alors une tendance ascendante depuis la moitié du XIXe siècle. Cette tendance favorable à la francophonie de la population tchèque se poursuivait au cours de la première moitié du XXe siècle, jusqu'en 1945. C'était lié aussi à la démocratisation des études secondaires qui étaient et sont toujours la base de la francophonie tchèque. Pourtant, en nombres absolus, il s'agissait tout au plus de quelques dizaines de milliers d'apprenants au tournant des $\mathrm{XI}^{\mathrm{e}}$ et $\mathrm{X} \mathrm{X}^{\mathrm{e}}$ siècles. L'essor le plus marquant de l'enseignement du français date de 1918-1939, où l'apprentissage du français concernait environ 68 \% des élèves des écoles secondaires tchèques en Bohême, Moravie et Silésie (Raková, 2011 : 41-61).

La chute rapide de l'apprentissage du français date de la période après 1945, lorsque le français cessa d'être matière obligatoire et figura dorénavant dans les programmes des écoles secondaires uniquement comme «langue étrangère», sur le même pied avec l'anglais et plus tard aussi avec l'allemand, expulsé temporairement des écoles tchèques entre 1945 et 1950. Entre 1945 et 1989, c'était le russe qui a pris la position de la première langue étrangère, longtemps réservée à l'allemand, dans tous les types d'écoles tchécoslovaques. À la suite des réformes scolaires socialistes, la part réservée à l'enseignement du français fut sensiblement réduite entre 1948 et 1989. La position du français se détériorait rapidement de 1948 à 1953, l'année où les derniers bacheliers des lycées passèrent le baccalauréat de français. Entre 1954 et 1963, le français cessa d'être matière au baccalauréat, ainsi que les autres langues occidentales. Le nombre de Tchèques francophones baissa et restait limité à un nombre très faible : 1000 élèves francophones dans tout le secondaire en 1956, contre au moins 34000 en 1921 (Raková, 2011 : 63-71, 135-138).

Ce n'est que dans les années 1960 que l'on assiste à une reprise de l'enseignement du français (dans le secondaire, dans les universités et à nouveau dans le primaire supérieur), ainsi que des rapports culturels et scolaires franco-tchèques et des possibilités de voyages touristiques et d'études en France. Mais les années 1970-1980 ont apporté un nouveau recul de l'enseignement du français. Depuis 1989, la francophonie de la population tchèque est à nouveau en augmentation. Le français est appris chaque année par un nombre croissant d'élèves tchèques. En 1990 et 1991, on a créé cinq lycées avec une section bilingue franco-tchèque, dispensant pendant les deux premières années l'enseignement intensif du français, suivi de quatre années pendant lesquelles cinq matières sont dispensées directement en français. Ces lycées bilingues franco-tchèques, de même que sept lycées avec des classes à l'enseignement élargi du français forment d'excellents 
francophones. Nous pouvons constater que malgré que la francophonie de la population tchèque ait toujours été acquise, donc le français n'a jamais été ni langue maternelle ni langue seconde de la population autochtone, il y a, et il y avait par le passé, des groupes de locuteurs tchèques qui l'utilisent comme langue de communication occasionnelle et comme langue de culture (Raková, 2011 : 72-82).

Si nous examinons maintenant le cas de la République tchèque par rapport à des autres Peco observateurs de l'OIF, nous constatons les mêmes effectifs faibles de locuteurs francophones (ou d'apprenants du français, ce qui revient au même dans le contexte de l'Europe centrale), un passé sociolinguistique commun (et notamment la prédominance de l'allemand et du russe en Europe centrale) qui n'était pas trop favorable à l'apprentissage du français dans une telle mesure comme en Europe occidentale où le français était longtemps une langue privilégiée des élites sociales et où il entre actuellement, depuis plusieurs décennies, en concurrence avec l'anglais notamment, mais aussi avec l'allemand, l'espagnol et l'italien.

La francophonie tchèque rentre tout à fait dans le cadre de la francophonie centreuropéenne. En témoigne le nombre de locuteurs francophones / francophones partiels en République tchèque $(2,4 \%)$, comparable avec celui des pays voisins (3\% en Pologne, $1,9 \%$ en Slovaquie, $0,9 \%$ en Hongrie) (La Langue française dans le monde 2010 : 14-15).

\section{Conclusion}

La famille francophone mondiale compte plus de 220 millions de francophones : on n'a jamais autant parlé le français comme à l'heure actuelle, au moins à l'échelle mondiale. En Europe, la situation de la diffusion du français semble moins brillante.

La spécificité de la francophonie européenne par rapport par exemple à la francophonie africaine consiste dans le paradoxe suivant : un nombre de plus en plus élevé de pays membres ou observateurs de l'OIF, mais les effectifs de francophones réels qui restent plutôt stables. De l'autre côté, plusieurs pays européens n'étant pas organisés dans l'OIF ont cependant des potentiels forts en nombre d'apprenants du français.

Le taux relativement faible de locuteurs francophones en République tchèque, comparable avec celui d'autres pays Peco faisant partie de l'OIF, est dû à des causes à la fois historiques et politiques. Les raisons historiques résultent de la réalité politique et sociolinguistique des Pays tchèques, ayant fait partie jusqu'en 1918 de l'État multinational des Habsbourgs (ce qui explique la prédominance traditionnelle de l'allemand dans l'éducation scolaire) et ensuite, de 1945 jusqu' en 1989, étant sous l'influence politique de l'URSS (ce qui explique la prédominance du russe parmi les langues étrangères enseignées). Les raisons politiques de la place actuelle du français en République tchèque coïncident avec la politique linguistique adoptée par le gouvernement tchèque. Actuellement, deux langues étrangères sont obligatoires à partir de l'école primaire (LV1 de la troisième classe et LV2 de la septième classe de l'école fondamentale), elles le sont également dans les lycées d'enseignement général mais pas dans les lycées techniques, où une seule langue est obligatoire. Les plans d'études récents, en vigueur depuis 2008, accordent à l'anglais la place privilégiée de LV1 (de facto, pas de iure). Le français qui se partage la place de LV2 avec l'allemand, l'espagnol, le russe, l'italien est actuellement au troisième rang par nombre d'apprenants. La situation en République tchèque au niveau des politiques linguistiques est comparable à celle de la plupart des pays de l'UE (deux langues vivantes obligatoires pour la plupart des élèves et le français choisi comme LV2 ou LV3). 
La francophonie européenne et tchèque cent trente ans après l'invention du terme par Onésime Reclus

Avec au moins 116 millions d'apprenants dans le monde, le français est l'une des seules langues à être enseignée dans tous les pays du monde. Il est incontestable qu'au niveau de l'Europe, la Francophonie a besoin de nouveau apprenants du et en français, notamment dans les Peco, mais aussi dans tous les pays n'ayant pas des pourcentages élevés des francophones natifs. Sans ses apprenants (et professeurs), la Francophonie institutionnelle deviendrait bientôt une organisation privée de sa raison d'être puisqu'il n'y aurait plus de locuteurs francophones qu'elle est censée regrouper par des liens de solidarité, à l'échelle planétaire.

Si nous comparons la situation actuelle de la francophonie mondiale et européenne avec celle de l'époque du fondateur de la notion de francophonie, Onésime Reclus, nous constatons un progrès incontestable du nombre de locuteurs francophones à l'échelle mondiale. En Europe, les francophones de l'OIF étaient plus de deux fois plus nombreux en 2010 qu'en 1880, en Afrique, les francophones de l'OIF se sont multipliés presque 13 fois par rapport à 1900 et 28 fois par rapport à 1880, en Asie, les francophones de l'OIF sont actuellement 30 fois plus nombreux que n'étaient les francophones de ce continent en 1880, et en Amérique, le nombre des francophones de l'OIF étaient en 2010 presque quatre fois supérieur à celui de 1900 et cinq fois supérieur à celui de 1880 .

Les chiffres absolus sont très optimistes mais ne rendent pas compte de la situation du français d'une manière complexe, puisqu'en fait, l'importance relative du français entre 1900 et 2010 a diminué.

Il s'avère nécessaire de faire augmenter les effectifs d'apprenants du et en français si l'ont veut maintenir la vivacité de la F/francophonie européenne et tchèque. Nous proposons deux solutions pour faire croître le nombre d'apprenants du et en français : la sensibilisation des élèves et de leurs parents pour qu'ils optent pour le français aussi comme LV1, non seulement comme LV2 ou LV3, et le changement des politiques scolaires en matière de l'enseignement de langues, concrètement en faveur de l'introduction de trois langues vivantes obligatoires au sein de l'Union européenne.

\section{Bibliographie}

CHAUdENSON, Robert (2006), Vers une autre idée et pour une autre politique de la langue française, Paris : L'Harmattan.

La Langue française dans le monde 2010 (2010), Paris : Nathan. Ouvrage réalisé par l'Observatoire de la langue française de l'OIF. Préfacé par Abdou Diouf.

Montenay, Yves (2005), La langue française face à la mondialisation, Paris : Les Belles Lettres. Préfacé par Antoine Sfeir.

Poissonnier, Ariane, Sournia, Gérard (2006), Atlas mondial de la francophonie, Paris : Éditions Autrement.

RaKovÁ, Zuzana (2011), Francophonie de la population tchèque 1848-2008, Brno : Masarykova Univerzita.

ReZnIKow, Stéphane (2002), Francophilie et identité tchèque (1848-1914), Paris : H. Champion.

Wolton, Dominique (2006), Demain la Francophonie. Pour une autre mondialisation, Paris : Flammarion.

\section{Sitographie}

http://www.ined.fr/fr/pop_chiffres/pays_du_monde/, le 30/07/2011.

http://www.francophonie.org/, le 30/07/2011. 
http:/ / afriessence.canalblog.com/archives/2006/12/07/3368831.html, le 30/07/2011.

http:/ / www.ladocumentationfrancaise.fr/dossiers/francophonie/onesimereclus.shtml, le 30/07/2011

http:/ / www.ladocumentationfrancaise.fr/dossiers/francophonie/peresfrancophonie.shtml, le 30/07/2011.

http:/ / www.francophonie.org/IMG/ pdf/Synthese-Langue-Francaise-2010.pdf, le $14 / 01 / 2011$.

Zuzana Raková

Ústav románských jazyků a literatur

Filozofická fakulta

Masarykova Univerzita v Brně

Arna Nováka 1

60200 Brno

République tchèque

Rakovaz@seznam.cz 